EPJ Web of Conferences 49, 18003 (2013)

DOI: 10.1051 /epjconf/20134918003

(C) Owned by the authors, published by EDP Sciences, 2013

\title{
Production and decay of a heavy radion in Randall-Sundrum model at the LHC
}

\author{
Yoshiko Ohno ${ }^{1}$ and Gi-Chol Cho ${ }^{2}$ \\ ${ }^{1}$ Graduate School of Humanities and Sciences, Ochanomizu University, Tokyo 112-8610, Japan \\ ${ }^{2}$ Department of Physics, Ochanomizu University, Tokyo 112-8610, Japan
}

\begin{abstract}
We study production and decay of a radion predicted in the Randall-Sundrum model at the LHC. The radion is a scalar particle and its production and decay channels are quite similar to those of the Higgs boson. We find constraints on the model parameter space taking account of the results of the Higgs boson search at the LHC. We also study a possibility discriminating the radion from the scalar particles in the other model such as the supersymmetric standard model at the LHC experiments.
\end{abstract}

\section{Introduction}

A warped extra dimension model proposed by Randall and Sundrum (RS) [1] is one of the promissing candidates of physics beyond the Standard Model (SM) to solve the gauge hierarchy problem. The RS model is formulated in the five-dimensional anti de-Sitter $\left(\mathrm{AdS}_{5}\right)$ space-time, and the extra (fifth) dimension is compactified on an orbifold $S_{1} / Z_{2}$ of a radius $r_{c}$. In the RS model, there are two 3-branes located at two orbifold fixed points on the coordinate of the fifth dimension $y=0$ and $y=\pi r_{c}$. The former is called a hidden brane while the latter is called a visible brane. All the SM particles are confined in the visible brane and only the gravity is allowed to propagate into the fifth dimension. With this set-up, the hierarchy between the electroweak scale and the Planck scale is understood reasonably if the distance between two 3-branes (i.e., compactification radius $r_{c}$ ) and the AdS curvature $(k)$ satisfies a condition, $k r_{c}=10 \sim 12$. It is, therefore, necessary to stabilize the radius of the extra dimension to solve the gauge hierarchy problem. A simple stabilization mechanism of the distance between two branes is known as the Goldberger-Wise mechanism [2].

A radion is a scalar field which corresponds to the fluctuation of the distance between two 3-branes. After the stabilization of the compactification scale, the radion has a finite mass and couples to the trace of the energymomentum tensor of the SM. As a result, production and decay channels of radion at the hadron collider are the same with those of Higgs boson in the SM.

In this presentation, we show results of our study about production and decay of the radion at the LHC. We find constraints on the mass and coupling of the radion from the results of the Higgs boson search at the LHC. We also examine a possibility to discriminate the radion from heavier Higgs bosons in the minimal supersymmetric standard model (MSSM). We found that, if the mass spectrum of
Higgs bosons in the MSSM follows so called the "decoupling scenario", the decay channels into the weak gauge boson pair ( $W W$ and $Z Z$ ) are useful channels to distinguish the radion in the RS model from the heavier Higgs bosons in the MSSM at the LHC.

\section{Production and decay of the radion}

The radion couples to the trace of the energy-momentum tensor of the SM. The interactions of radion with SM fields are described by the following Lagrangian:

$$
\mathcal{L}_{\mathrm{int}}=\frac{\phi}{\Lambda_{\phi}}\left\{T_{\mu}^{\mu}(\mathrm{SM})+T_{\mu}^{\mu}(\mathrm{SM})^{\mathrm{anom}}\right\}
$$

where $\phi$ is the radion field and $\Lambda_{\phi}$ is its vacuum expectation value $(\mathrm{VEV})$. The trace of energy-momentum tensor $T_{\mu}^{\mu}(\mathrm{SM})$ is given by

$$
\begin{gathered}
T_{\mu}^{\mu}(\mathrm{SM})=\sum_{f} m_{f} \bar{f} f-2 m_{W}^{2} W_{\mu}^{+} W^{-\mu}-m_{Z}^{2} Z_{\mu} Z^{\mu} \\
+\left(2 m_{h}^{2} h^{2}-\partial_{\mu} h \partial^{\mu} h\right)+\cdots
\end{gathered}
$$

while $T_{\mu}^{\mu}(\mathrm{SM})^{\text {anom }}$ comes from the scale anomaly

$$
T_{\mu}^{\mu}(\mathrm{SM})^{\mathrm{anom}}=\sum_{a} \frac{\beta_{a}\left(g_{a}\right)}{2 g_{a}} F_{\mu \nu}^{a} F^{a \mu \nu},
$$

where $a, \beta_{a}$ and $F_{\mu \nu}^{a}$ are the gauge index, the $\beta$-function and the field strength for a corresponding gauge group, respectively (for detail, see [3, 4]).

From eqs. (1), (2) and (3), we can calculate the decay rate of the radion into the SM fermions, gauge bosons and the Higgs boson. In Figure 1, we show the branching ratios of the radion decaying to the various SM particles as a function of the radion mass $m_{\phi}$. The Higgs boson mass $m_{h}$ in the final state is fixed at $125 \mathrm{GeV}$. Note that the dominant decay modes of radion are the $W W, Z Z$ and $h h$ channels for $m_{\phi} \geq 500 \mathrm{GeV}$. 


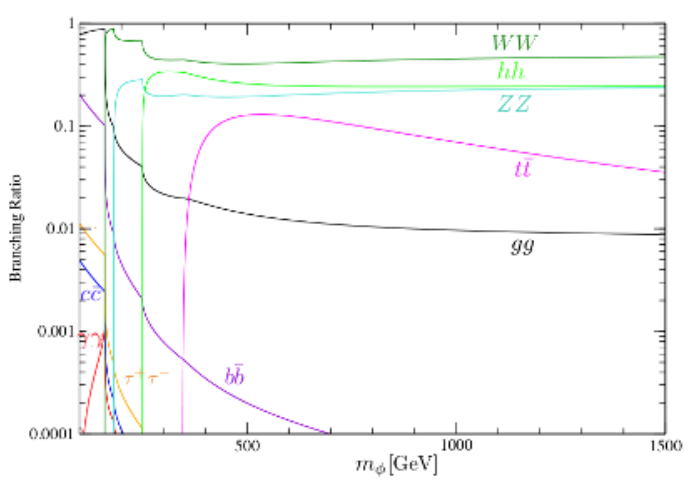

Figure 1. Branching ratio of the radion decaying to the SM particles as a function of the radion mass $m_{\phi}$. For a decay to the Higgs boson pair, $\phi \rightarrow h h$, the Higgs boson mass is fixed at $125 \mathrm{GeV}$.

\section{Constraints on the radion mass}

The RS model is characterized by two parameters, $m_{\phi}$ and $\Lambda_{\phi}$. Constraints on the parameter $\Lambda_{\phi}$ has been studied indirectly using the result of the 1st Kaluza-Klein graviton search at the LHC [5]. As mentioned in a previous section, the decay modes of the radion are completely same with those of the SM Higgs boson up to the couplings. In this section, therefore, we study constraints on $m_{\phi}$ and $\Lambda_{\phi}$ using the experimental data of the Higgs boson search at the LHC.

At the LHC, a signal of Higgs-like particle has been found at about $125 \mathrm{GeV}$ in both ATLAS and CMS, and the wide mass region except for $\sim 125 \mathrm{GeV}$ has been excluded. For example, the ATLAS experiment excludes the mass smaller than $558 \mathrm{GeV}$ from the decay channels $h \rightarrow W W$ and $h \rightarrow Z Z$ [6]. This mass bound may be understood as a lower mass bound on an scalar particle in some new physics models if the scalar particle couples to $W W$ and $Z Z$. Since the radion also decays into both $W W$ and $Z Z$, we examine constraints on the mass of the radion $m_{\phi}$ and the coupling $\Lambda_{\phi}$ from the results of ATLAS experiment. In the following, we study the allowed parameter space of the radion under the condition

$$
\begin{aligned}
& \sigma(p p \rightarrow h) \times\left.\operatorname{Br}(h \rightarrow W W / Z Z)\right|_{m_{h}=558 \mathrm{GeV}} \\
& \geq \sigma(p p \rightarrow \phi) \times \operatorname{Br}(\phi \rightarrow W W / Z Z) .
\end{aligned}
$$

We show constraints on $m_{\phi}$ and $\Lambda_{\phi}$ taking account of the condition (4) in Figure 2. Two contours correspond to the $95 \%$ CL bounds obtained from the Higgs search limit in the $W W$ channel (red) and the $Z Z$ channel (blue), respectively. The inner of contours are excluded region. It can be seen from the figure that the radion mass $m_{\phi}$ should be larger than $500 \mathrm{GeV}$ for $\Lambda_{\phi}<5 \mathrm{TeV}$. As $\Lambda_{\phi}$ increases, bound on $m_{\phi}$ is weakened. Note that constraints from $h \rightarrow Z Z$ are much stronger than those from $h \rightarrow W W$. For example, $m_{\phi}=300 \mathrm{GeV}$ and $\Lambda_{\phi}=8 \mathrm{TeV}$ is strongly disfavored by the experimental bounds for $h \rightarrow Z Z$ (not by $h \rightarrow W W$ ) at the LHC. However, the sensitivity of the $W W$ mode for small $m_{\phi}$ is slightly better than $Z Z$ mode.
From this result, we conclude that the radion lighter than $\sim 300 \mathrm{GeV}$ is disfavored from the Higgs search experiment at the LHC unless the parameter $\Lambda_{\phi}$ is larger than $10 \mathrm{TeV}$. Since too large $\Lambda_{\phi}$ is unfavorable because it may lead to a new hierarchy between the weak scale and $\Lambda_{\phi}$, we focus on the heavy radion search at the LHC.

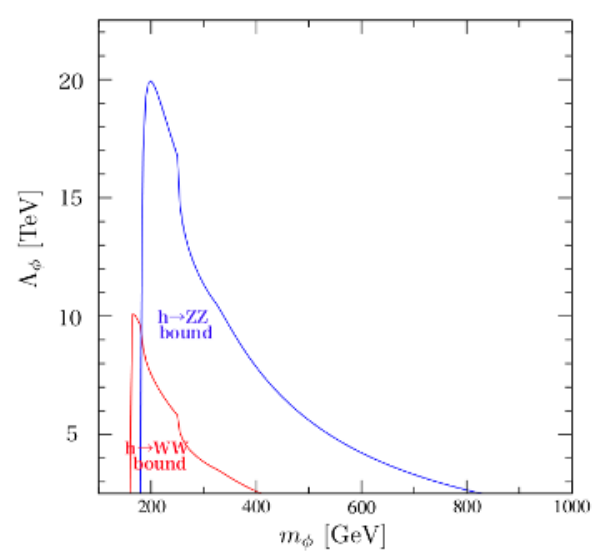

Figure 2. Constraints on $m_{\phi}$ and $\Lambda_{\phi}$ from the results of the Higgs search experiment at the LHC. The red and blue contours are obtained from the experimental bounds on the decay channels $h \rightarrow W W$ and $h \rightarrow Z Z$, respectively.

\section{Radion vs. MSSM Higgs}

Once a new scalar particle beyond the SM is found in the future LHC experiments, we must study if it is the radion or not, because various models predict an existence of neutral scalar particles. In this section, we examine a possibility to discriminate the radion from the heavier Higgs bosons in the MSSM as an example. In the MSSM, there are five Higgs bosons - two $C P$-even scalars $(h, H)$, one $C P$-odd scalar $(A)$ and two charged scalars $\left(H^{ \pm}\right)$. It is known that the mass of scalar particles discovered at the $\mathrm{LHC}, \sim 125 \mathrm{GeV}$, is very close to the upper limit of the lightest Higgs boson $h$ in the MSSM. A possible explanation on the mass of $h$ is known as so called "decoupling scenario" where the lightest Higgs boson $h$ has almost same property with the SM while the other four Higgs bosons are much heavier than $h$ and degenerate in mass. In the following, we identify the lightest Higgs boson $h$ as a particle discovered at the LHC and compare the production and decay of the heavy neutral Higgs bosons $(H, A)$ with those of the radion.

Figure 3 shows the production cross sections of the radion $\phi$ and MSSM Higgs bosons $H, A$ at the LHC as functions of their mass. The dashed lines represent the radion with various values of the parameter $\Lambda_{\phi}$ from $2.5 \mathrm{TeV}$ to $15 \mathrm{TeV}$. The solid lines in red and in cyan correspond to $H$ and $A$, respectively. The cross sections of $H$ and $A$ are obtained for $\tan \beta=30$ as an example, where $\tan \beta$ is a ratio of VEV of two Higgs doublets. It is easy to see that, when the mass is larger than $500 \mathrm{GeV}$, the production cross section of the radion is much larger than those of $H$ and $A$ for $\Lambda_{\phi} \leq 5 \mathrm{TeV}$. 


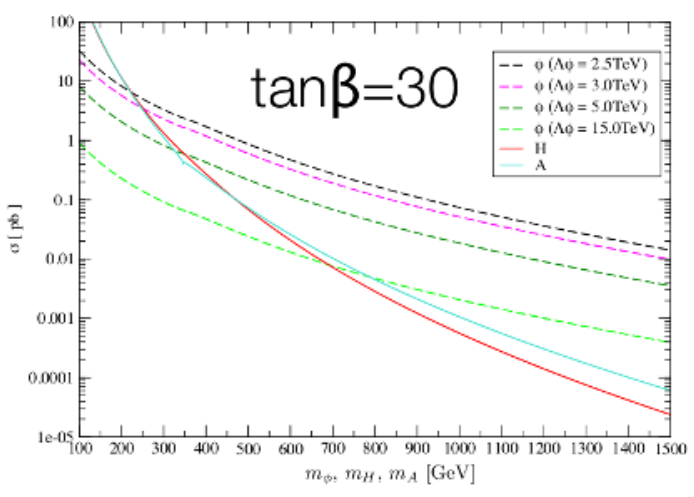

Figure 3. Production cross sections of $\phi, H, A$ as functions of their mass.

A distinctive feature of the decoupling scenario can be found in the interactions of the Higgs bosons. The interactions of the heavier Higgs bosons $H, A$ to the weak gauge bosons $W, Z$ are proportional to $\cos (\alpha-\beta)$, where $\alpha$ is the mixing angle of the mass matrix of the $C P$-even Higgs bosons. The decoupling scenario is realized when $\beta \simeq \alpha+\pi / 2$ so that the interactions of $H$ and $A$ to $W W$ and $Z Z$ are highly suppressed. On the other hand, the interactions of radion to $W W$ and $Z Z$ have no suppression like $H$ and $A$. We, therefore, expect relatively larger decay rates of the radion to $W W$ and $Z Z$ as compared to those of $H$ and $A$, and these decay modes could be promissing channels to distinguish the radion from the MSSM Higgs bosons.

We calculated the number of events $N$ of the processes $p p \rightarrow \phi \rightarrow W W / Z Z$ at the LHC with the center-ofmass energy $\sqrt{s}=7 \mathrm{TeV}$ and the integrated luminosity $\int d t \mathcal{L}=15 \mathrm{fb}^{-1}$. The results are shown in Table 1 for $\Lambda_{\phi}=8 \mathrm{TeV}$ and $15 \mathrm{TeV}$. In the table, the number of events of the $\phi \rightarrow Z Z$ mode is absent for $m_{\phi}=200 \mathrm{GeV}$. This is because that the radion mass $m_{\phi}=200 \mathrm{GeV}$ is disfavored from the search of Higgs boson in the $h \rightarrow Z Z$ channel at the LHC as shown in Figure 2. We obtain the number of events more than a thousand (a few hundred) for $m_{\phi}=200 \mathrm{GeV}$ and $\Lambda_{\phi}=8 \mathrm{TeV}(15 \mathrm{TeV})$. Even for larger mass, $m_{\phi}=500 \mathrm{GeV}$, we obtain $O(10)$ events for both $\Lambda_{\phi}=8 \mathrm{TeV}$ and $15 \mathrm{TeV}$ in both the $W W$ and $Z Z$ channels. Note that, as already discussed, the decays into $W W$ and $Z Z$ of heavier Higgs bosons $(H$ and $A$ ) in the decoupling scenario of MSSM are strongly suppressed. Thus, when a heavy and neutral scalar particle is discovered in the future
LHC experiment, we can expect to test a possibility of the radion using the $W W$ and $Z Z$ channels.

\section{Summary}

We have shown the results of our recent study for the production and decay of the radion in the RS model at the LHC. We first found constraints on the radion mass $m_{\phi}$ and the coupling $\Lambda_{\phi}$ from the experimental results of search for the Higgs boson at the LHC, since the radion has same decay channels with the Higgs boson. We examined a possibility to distinguish the radion from the other scalar particles such as the heavier Higgs bosons in the MSSM. The discovery of the Higgs-like particle at the LHC tells us that the decoupling scenario on the Higgs boson masses in the MSSM is one of the possible scenarios of the MSSM. We discussed that the decays into $W W$ and $Z Z$ are promissing channels to discriminate the radion from the heavier Higgs bosons $H, A$ in the decoupling scenario of the MSSM. When the radion mass is $500 \mathrm{GeV}$, a few $\times 10$ events are expected in the radion decays to $W W$ and $Z Z$ while decays of $H$ and $A$ into those final states are highly suppressed in the decoupling scenario of the MSSM.

The work of G.C.C is supported in part by Grantsin-Aid for Scientific Research from the Ministry of Education, Culture, Sports, Science and Technology (No.24104502) and from the Japan Society for the Promotion of Science (No.21244036).

\section{References}

[1] L. Randall and R. Sundrum, Phys. Rev. Lett. 83, 3370 (1999) [hep-ph/9905221].

[2] W. D. Goldberger and M. B. Wise, Phys. Rev. Lett. 83, 4922 (1999) [hep-ph/9907447].

[3] C. Csaki, J. Hubisz and S. J. Lee, Phys. Rev. D 76, 125015 (2007) [arXiv:0705.3844 [hep-ph]].

[4] K. -m. Cheung, Phys. Rev. D 63, 056007 (2001) [hep$\mathrm{ph} / 0009232]$.

[5] Y. Tang, arXiv:1204.6145 [hep-ph].

[6] G. Aad et al. [ATLAS Collaboration], Phys. Lett. B 717, 29 (2012) [arXiv:1205.6744 [hep-ex]].

[7] A. Djouadi, Phys. Rept. 459, 1 (2008) [hep$\mathrm{ph} / 0503173]$.

\begin{tabular}{llll}
\hline$m_{\phi}$ & $200 \mathrm{GeV}$ & $500 \mathrm{GeV}$ & $1 \mathrm{TeV}$ \\
$\Lambda_{\phi}=8 \mathrm{TeV}$ & & & \\
\hline$\sigma \times \operatorname{Br}(\phi \rightarrow W W)$ & $1.9 \times 10^{3}$ & $6.6 \times 10$ & 3.2 \\
$\sigma \times \mathrm{Br}(\phi \rightarrow Z Z)$ & & $3.2 \times 10$ & 1.6 \\
\hline \hline$\Lambda_{\phi}=15 \mathrm{TeV}$ & & & \\
\hline$\sigma \times \operatorname{Br}(\phi \rightarrow W W)$ & $5.4 \times 10^{2}$ & $1.9 \times 10$ & $9.0 \times 10^{-1}$ \\
\hline$\sigma \times \operatorname{Br}(\phi \rightarrow Z Z)$ & & 9.0 & $4.6 \times 10^{-1}$ \\
\hline
\end{tabular}

Table 1. Number of events of the radion decay $\phi \rightarrow W W / Z Z$ at $\sqrt{s}=7 \mathrm{TeV}$ for the integrated luminosity $15 \mathrm{fb}^{-1}$. 